\title{
Loxoscelismo dermonecrotico en bovinos
}

\author{
Dermonecrotic loxoscelism in bovine
}

Cardona-Álvarez JÁ, Lopez-Galvis CJ, Reyes-Bossa BJ, Loxoscelismo dermonecrotico en bovinos. Rev Colombiana Cienc Anim. Recia. 2020; 12(2):e788. https://doi.org/10.24188/recia.v12. n2.2020.788

Universidad de Sucre, Colombia

Los autores permiten a RECIA reimprimir el material publicado en él. En caso de que un autor quiera traducir o usar una publicación parcial o completa de nuestro Diario, el autor debe obtener un permiso por escrito del editor de la revista.

Copyright (C) 2020. El (los) autor (es), Revista Colombiana de Ciencia Animal - RECIA. 2020. Este es un artículo de acceso abierto distribuido bajo los términos de Creative Commons Attribution 4.0 (https://creativecommons.org/licenses/by-nc-sa/4.0/), El uso, distribución o reproducción está permitido, siempre que se acrediten al autor original y al propietario del copyright y que se cite la publicación original en esta revista, de acuerdo con la práctica académica aceptada. No se permite el uso, distribución o reproducción que no cumpla con estos términos. 


\title{
Loxoscelismo dermonecrotico en bovinos
}

\author{
Dermonecrotic loxoscelism in bovine
}

José Alberto Cardona Álvarez, Dr.Vet.Sc.

Universidad de Córdoba, Departamento de Ciencias Pecuarias, Grupo

DOI: https://doi.org/10.24188/recia.v12.n2.2020.788 de Investigaciones en Medicina de Grandes Animales (MEGA), Profesor Titular de Medicina y Clínica de Grandes Animales, Montería, Colombia. jacardonaalvarez@correo.unicordoba.edu.co

(iD) https://orcid.org/0000-0002-8254-1120

Carlos José Lopez Galvis, MVZ.

ICA, Extensionista Rural, Montería, Colombia

carlopezz2211@hotmail.com

(D) https://orcid.org/0000-0002-0801-3274

Bernardo José Reyes Bossa, M.Sc.

Universidad de Córdoba, Facultad de Medicina Veterinaria y Zootecnia, Grupo de Investigación en Medicina de Grandes Animales (MEGA), Montería, Colombia

breyesbossa@correo.unicordoba.edu.co

(i) https://orcid.org/0000-0002-8730-0350

Recepción: 12 mayo 2020

Aprobación: 25 Agosto 2020

Publicación: 17 Septiembre 2020

\section{RESUMEN}

Fueron atendidos por el Servicio Clínico Ambulatorio de Grandes Animales de la Facultad de Medicina Veterinaria y Zootecnia de la Universidad de Córdoba, dos bovinos hembra, ambas de la raza Brahman, una novilla de 15 meses de edad y un peso de $150 \mathrm{Kg}$ y una vaca de 5 años y un peso $400 \mathrm{~kg}$, ambos casos ubicados en el municipio de Cereté, Córdoba. La anamnesis indica que la novilla de 15 meses comenzó mostrando una leve hinchazón cutánea nivel de la región abdominal ventral y pectoral, con un progresivo aumento de volumen del área afectada durante una semana, de igual forma, la vaca de 5 años comenzó con enrojecimiento de la piel de la glándula mamaria y del pezón. Se realizó un examen clínico general y específico del sistema tegumentario, donde se analizaron las características de la lesión, así como los posibles diagnósticos presuntivos, basado en la forma, ubicación, extensión, humedad y profundidad de la lesión; y de las condiciones epidemiológicas de la zona. En ambos animales, las lesiones fueron en común caracterizadas por una necrosis progresiva, con una zona eritematosa y edematosa, presencia de un punto necrótico central y un halo medial blanquecino y uno más externo violáceo, hiperestesia de la zona. Se concluye la importancia de conocer la epidemiología de las alteraciones dermatológicas en bovinos del Departamento de Córdoba, así como, el buen uso del examen clínico, la caracterización de las lesiones y la presencia de la araña en las explotaciones bovinas donde se presenten casos de dermonecrósis para el correcto diagnóstico de loxoscelismo.

Palabras clave: Loxosceles laeta; dermonecrósis; accidente ponzoñoso; bovinos.

\begin{abstract}
Two female bovines, both of the Brahman breed, a 15-month-old heifer weighing $150 \mathrm{Kg}$ and a cow were treated by the Outpatient Clinical Service of Large Animals of the Faculty of Veterinary Medicine and Zootechnics of the University of Córdoba. 5 years old and weighing $400 \mathrm{~kg}$, both cases located in the municipality of Cereté, Córdoba. The anamnesis indicates that the 15-month-old heifer began showing a slight cutaneous swelling at the ventral abdominal and pectoral region, with a progressive increase in volume of the affected area during a week, in the same way, the 5-year-old cow began with redness of the skin of the mammary gland and nipple. A general and specific clinical examination of the integumentary system was carried out, where the characteristics of the lesion were analyzed, as well as the possible presumptive diagnoses, based on the shape, location, extension, humidity and depth of the lesion; and the epidemiological conditions of the area. In both animals, the lesions were characterized in common by progressive necrosis, with an
\end{abstract}


erythematous and edematous area, the presence of a central necrotic spot and a whitish medial halo and a purplish outer halo, hyperesthesia in the area. The importance of knowing the epidemiology of the dermatological alterations in cattle in the Department of Córdoba is concluded, as well as the good use of the clinical examination, the characterization of the lesions and the presence of the spider in the bovine farms where cases of dermonecrosis occur. for the correct diagnosis of loxoscelism.

Keywords: Loxosceles laeta; dermonecrosis; poisonous accident; bovines.

\section{INTRODUCCIÓN}

Existen más de 40,000 especies de arañas, probablemente 100.000 por describir, pero solo 3 taxones son reconocidos como peligrosos, a saber, Theridiidae, Loxoscelidae y Ctenidae (1).

La araña Loxosceles spp., es la única especie arácnida de distribución cosmopolita que es capaz de producir una lesión dermonecrótica en el sitio de la picadura; sin embargo, en algunos casos puede llegar a producir manifestaciones sistémicas, hemólisis y falla renal subsecuente, hasta provocar la muerte del animal (2). El primer caso documentado de loxoscelismo fue en 1879 en Tennessee en (1).

El género Loxosceles incluye diferentes especies de arañas que representan una causa importante de aracnidismo necrótico, la araña marrón, como es llamada vulgarmente, puede medir entre 1 a $5 \mathrm{~cm}$ de pierna a pierna, es de color marrón oscuro y tiene en su cefalotórax dorsal una marca en forma de violín color marrón, no hay evidencia de que esta araña sea agresiva, al contrario, la araña tiende a habitar áreas oscuras y retiradas, aunque la araña ha llevado su habitad hasta zonas urbanas, como casas, garajes entre otros, donde su encuentro con humanos torna a ser más frecuente (3). Las Loxosceles, son arañas de actividad principalmente nocturna, se reproducen fundamentalmente en la época de verano, se alimentan de pequeños insectos (4).

Loxoscelismo, se define como un cuadro producido por el veneno de las arañas del género Loxosceles (Loxos: curvas, kelos: patas) recibe nombres como araña de rincón, araña parda, marrón, viuda marrón o violinista (5). En Colombia, no existen datos epidemiológicos que permitan evidenciar la incidencia anual; sin embargo, el loxoscelismo se presenta con más frecuencia en áreas rurales, desde 2800 msnm hasta el nivel del mar (2).

Se tiene registro de cuatro géneros de arañas con capacidad de inocular veneno en Colombia: Género Theraphosa (tarantulas), género Latrodectus (viuda negra), género Phoneutria (araña bananera), y género Loxosceles (arañas violinistas, de rincón o reclusa); de los cuales sólo el género Loxosceles es capaz de producir este tipo de lesiones dermonecróticas (6). En Colombia se conocen las especies Loxosceles rufipes, Loxosceles rufescens y Loxosceles laeta. Se consideran animales ponzoñosos y de gran importancia médica en las áreas rurales, debido a que poseen quelíceros para inyectar el veneno (2).

"El veneno de las arañas Loxosceles spp., es una mezcla de toxinas proteicas enriquecidas con moléculas de baja masa molecular (5-40 kDa), varias toxinas han sido identificadas y bien caracterizado bioquímicamente. Estas incluyen fosfatasa alcalina, ribonucleótido fosfohidrolasa, hialuronidasa, serina proteasas, metaloproteasas y esfingomielinasa-D, Metaloproteasas llamadas Loxolysin A (20-28 kDa) y Loxolysin B (32-35 kDa) tienen actividad gelatinolitica, fibronectinolitica y fibrinogenolíticas y pueden desempeñar un papel en alteraciones hemostáticas que ocurren después de envenenamiento como lesión de vasos sanguíneos, hemorragia en el dermis, adherencia plaquetaria imperfecta y defectuosa cicatrización de la herida; la toxina hialuronidasa degrada residuos de ácido hialurónico y sulfato de condroitina de proteoglicanos y podría ser putativamente involucrado en el diseminación gravitacional de la dermonecrosis y como factor de propagación sistémico; la esfingomielinasa-D (30-35 kDa), también llamada toxina dermonecrótica, es la molécula mejor caracterizada bioquímicamente, identificada en veneno de diferentes especies de Loxosceles. Esta toxina, como molécula nativa o como variantes recombinantes, puede inducir dermonecrosis, agregación plaquetaria y hemolisis experimental. De igual forma, contribuye directa o indirectamente a actividades citotóxicas en diferentes células, tiene actividad hemolítica en los eritrocitos, y causa la agregación plaquetaria. In vivo parece tener un efecto importante en la inhibición de la quimiotaxis de neutrófilos (7).

El loxoscelismo puede presentarse de dos formas distintas: cuadro cutáneo y cuadro sistémico. El desarrollo de uno de los cuadros o de ambos y la gravedad de los cuadros depende de diversos factores relacionados tanto al accidentado como a las características de la araña, ejemplos de estos factores son: cantidad de veneno inoculado, estado nutricional y edad de la víctima, así como la región de la picadura, así como especie, sexo y estado de desarrollo de la araña (8). 
El compromiso cutáneo necrótico, es el más frecuente y corresponde a un cuadro circunscrito que puede ir desde una zona eritematosa pequeña hasta una extensa área de necrosis dérmica con ulceración posterior. El dolor es inmediato y progresivo y existe una marcada hiperestesia peri-lesional, edema y eritema de la zona y un halo vaso constrictivo azul grisáceo alrededor del sitio de la mordedura (uno blanquecino medial y uno violáceo más externo). El eritema es reemplazado por una decoloración violácea característica de la piel llamada placa "livedoide" que progresa a una lesión necrótica que se va circunscribiendo y cuyos bordes se van delimitando $(9,10)$, para en cuestión de días formarse una costra que posteriormente se desprende dejando una zona ulcerada que cicatrizará por segunda intención. Por el otro lado, el cuadro de loxoscelismo visceral se caracteriza por la presencia de compromiso sistémico, eventualmente letal, caracterizado por hemólisis, hematuria, hemoglobinuria, ictericia, fiebre e insuficiencia renal, que ocurre generalmente transcurridas las primeras 24 horas de la mordedura (11).

El tratamiento adecuado para el loxoscelismo es aun motivo de controversia. Algunas terapias reportadas incluyen oxígeno hiperbárico, dapsona, antihistamínicos, antibióticos, dextrano, glucocorticosteroides, vasodilatadores, heparina, nitroglicerina, escisión quirúrgica y suero anti-arácnido (5).

Son muy pocos y recientes los estudios sobre el loxoscelismo en animales de la costa norte colombiana, lo que constituye este trabajo como literatura de referencia para profesionales del área, cuyo objetivo fue describir las características de las lesiones clínicas en dos casos de loxoscelismo dermonecrótico en bovinos del departamento de Córdoba.

\section{REPORTE DE CASO}

Antecedentes. Fue atendido por el Servicio Clínico Ambulatorio de Grandes Animales de la Universidad de Córdoba, dos bovinos hembras, ambas de la raza Brahman, una novilla de 15 meses de edad y un peso de 150 Kg y una vaca de 5 años de edad y un peso $400 \mathrm{~kg}$, ambos casos ubicados en el municipio de Cereté, Córdoba.

Anamnesis. El operario de los animales reporta que la novilla de 15 meses comenzó mostrando una leve hinchazón cutánea nivel de la región abdominal ventral y pectoral, con un progresivo aumento de volumen del área afectada durante una semana (Figura 1). Así mismo, informa que la vaca de 5 años igualmente comenzó con enrojecimiento de la piel de la glándula mamaria y del pezón. Ambos animales fueron manejados medicamente con antibióticos como oxitetraciclina L.A y AINES como el flunixin de meglumina, sin observar mejoría post aplicación del tratamiento, por lo que el área afectada comenzó a mostrar lesión cutánea con coloración negruzca y olor fétido.

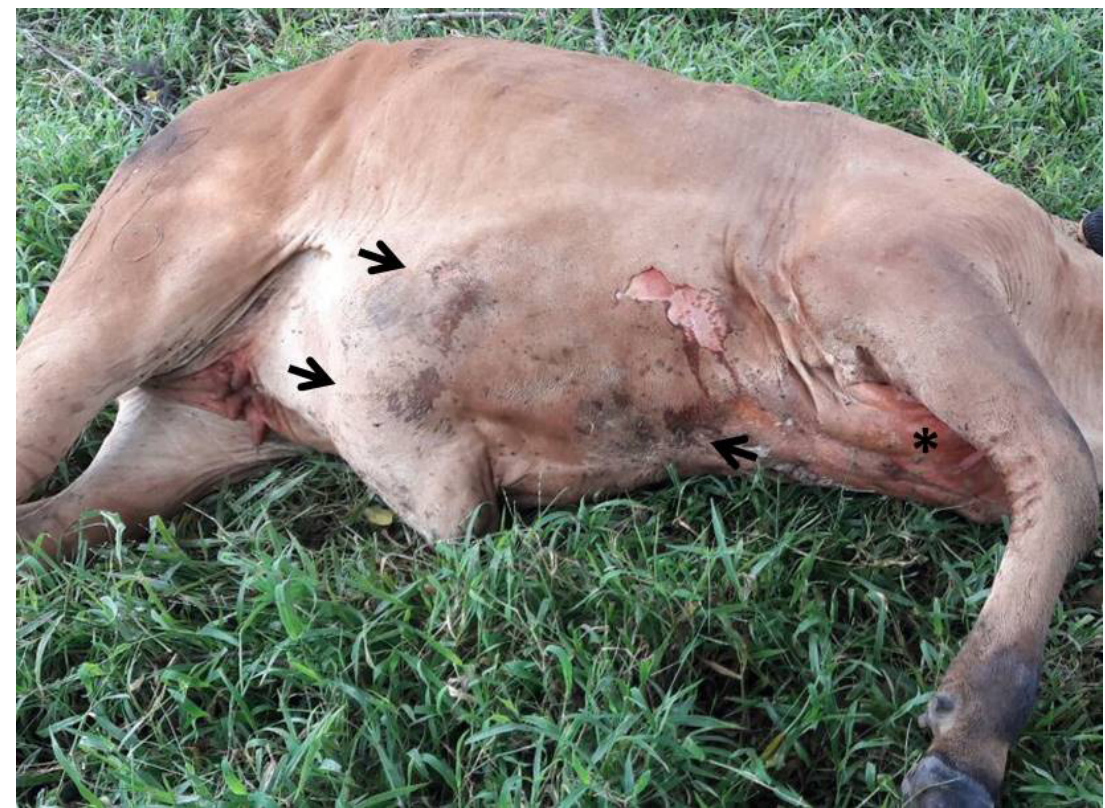

Figura 1. Región abdominal ventral y pectoral. Observe el marcado aumento de volumen de la zona (flechas) y algunas áreas de eritema (asterisco).

Se procedió a realizar la evaluación de la lesión previa sujeción física del animal siguiendo las las normas técnicas referentes a los principios éticos internacionales para la investigación biomédica con animales del CIOMS (Council for International Organizations of Medical Sciences) establecida por la UNESCO (United Nations Educational, Scientific and 
Cultural Organization) y la OMS (Organización Mundial de la Salud) de 1949 y de la ley 84 de octubre 27 de 1989 (Estatuto Colombiano de Protección Animal) (12).

Examen Físico. Se realizó un examen clínico general y específico del sistema tegumentario, donde se analizaron las características de la lesión, así como los posibles diagnósticos presuntivos, basado en la forma, ubicación, extensión, humedad y profundidad de la lesión; y de las condiciones epidemiológicas de la zona.

En ambos animales, las lesiones fueron en común caracterizadas por una necrosis progresiva, con una zona eritematosa y edematosa, presencia de un punto necrótico central y un halo medial blanquecino y uno más externo violáceo, hiperestesia de la zona. En la novilla de 15 meses, 8 días después de comenzado el cuadro, se evidenció formación de una costra apergaminada, de color negruzca, con posterior desprendimiento, dejando así una superficie ulcerada, con bordes violáceos, indicando necrosis progresiva (Figura 2). De igual forma, la vaca de 5 años, mostró aumento de tamaño de la glándula mamaria en el cuarto anterior izquierdo, así como engrosamiento del pezón del cuarto mamario afectado, evidenciando áreas negruzcas de dermonecrósis con un punto necrótico central y formación de un halo externo de color violáceo que corresponde al halo tóxico o progresivo (Figura 3).

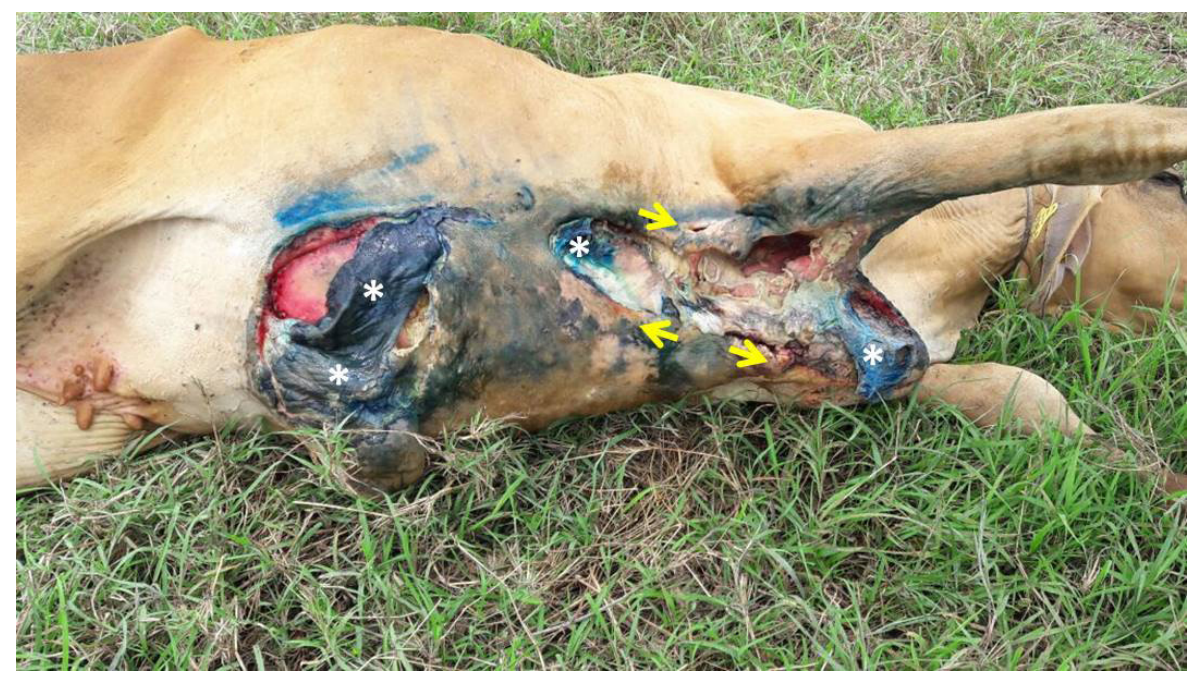

Figura 2. Loxoscelismo cutáneo en hembra bovina de 15 meses. Se observan áreas de necrosis central con desprendimiento de piel necrosada (asteriscos) y en algunas áreas se evidencia un halo violáceo oscuro más externo (flechas).

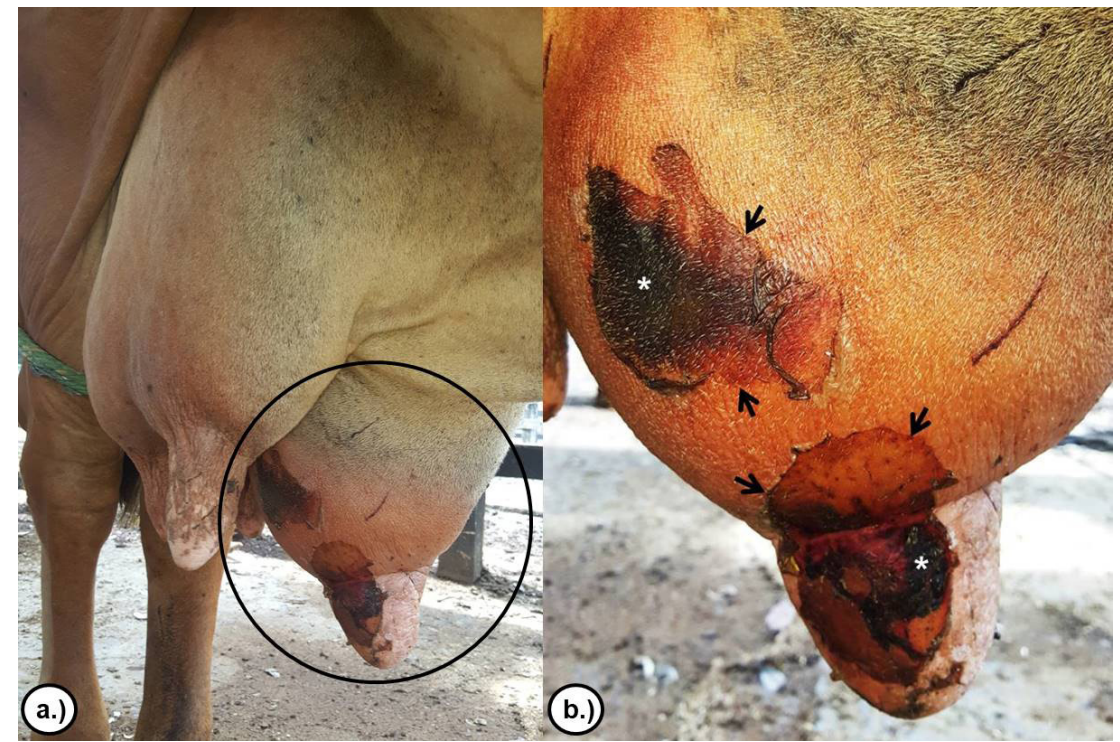

Figura 3. Dermonecrósis de la glándula mamaria y del pezón. a.) Observe el aumento de volumen del cuarto anterior izquierdo (círculo), con presencia de áreas focalizadas de necrosis. b.) Lesión dermonecrótica, se puede apreciar las áreas de necrosis (asterisco blanco) rodeado de un halo violáceo externo (flechas negras). 
La novilla por decisión del propietario fue eutanasiada, mientras que a la vaca adulta se le realizó tratamiento con glucocorticoides (dexametasona) a dosis de $0.08 \mathrm{mg}$ x kg durante 12 días, la cual presentó mejoría completa al finalizar el tratamiento.

\section{DISCUSIÓN}

Las características las lesiones, áreas de necrosis central con halo tóxico o progresivo, concuerdan con lo reportado por Herrera (9) y Espinosa et al (10). En su estudio, Violet et al (13), reportaron dos casos de loxoscelismo cutáneo en dos bovinos del Departamento de Córdoba, un macho Romo Sinuano y una hembra Brahman, encontrando características de la lesión muy parecidas en los animales, como edema y un área eritematosa focal, al detallar el área lesionada, se observó un punto necrótico central y dos halos alrededor (un halo blanco medial y un halo violáceo más externo), lo que ratifica el diagnóstico definitivo en el presente estudio.

Cardona et al (14), en un estudio realizado en equinos en el Departamento de Córdoba, reportaron características de las lesiones de animales con loxoscelismo dermonecrotico como: un punto necrótico central, dos halos alrededor (un halo blanco medial y un halo violáceo más externo) y la presencia de un área de necrosis progresiva, así mismo observaron en muestras histopatológicas teñidas con Hematoxilina-eosina, severa dermatitis piogranulomatosa, con marcada infiltración de polimorfonucleares especialmente neutrófilos, con vacuolización de la capa basal de la epidermis y edema en la unión dermo-epidérmica, indicando un proceso inflamatorio progresivo con la consiguiente necrosis.

Un estudio realizado en México, en 11 niños que habitaban en zonas rurales, se reportaron lesiones producidas por la araña Loxosceles spp., así mismo se informó dolor y eritema en el 100\% de los individuos, edema en un 82\%, necrosis en un $45 \%$ y fiebre en un $45 \%$ (15), lo que puede estar relacionado con la rápida atención médica que reciben los humanos al ser picados, de esta forma se puede concluir que de una rápida intervención médica en los animales, se pueden evitar lesiones necróticas graves.

El tratamiento utilizado para estos animales consistió en la aplicación de dexametasona (Azium ${ }^{\circledR}$, Schiring ploung, Colombia) a dosis de $0.08 \mathrm{mg}$ x kg cada 24 horas por 10 días, lo cual coincide con lo reportado por Violet et al (13) y Cardona et al. (14) como parte del manejo médico en su informe. De igual forma, coincidió con Roodt et al (16) Quienes usaron prednisona a dosis de 0.5 a $1 \mathrm{mg}$ x $\mathrm{kg}$ en humanos, aunque fue la misma molécula, ambas pertenecen a la familia de los corticosteroides. Así mismo, Cardona (17), recomienda para el manejo médico del loxoscelismo dermonecrótico, el uso de 3 medicamentos que interrumpen las vías fisiopatológicas, como son la dapsona, la dexametasona y la heparina de bajo peso molecular (enoxiheparina).

Se concluye la importancia de conocer la epidemiología de las alteraciones dermatológicas en bovinos del Departamento de Córdoba, así como, el buen uso del examen clínico, la caracterización de las lesiones y la presencia de la araña en las explotaciones bovinas donde se presenten casos de dermonecrósis para el correcto diagnóstico de loxoscelismo.

\section{REFERENCIAS}

1. Appel M, Bertoni R, Gremski W, Veiga S. Insights into brown spider and loxoscelism. ISJ. 2005; 2: 152-158.

2. Aguiar L, gonzáLez P, SarMiento K, SaLaManca N, Segura J, L Vargas. Accidente por araña Loxosceles spp.: reporte de caso y revisión de literatura. Univ. Méd. 2014; 55(3):340-348. https://doi.org/10.11144/Javeriana.umed55-3.aals

3. Malaque C, Castro J, Cardoso J, Fransa O, Barbaro A, Fan h. Clinical and epidemiological features of definitive and presumed loxoscelism in são paulo, brazil. Rev Inst Med Trop. 2002; 44(3):139-143. https://doi.org/10.1590/ S0036-46652002000300005

4. Hernandez N, Alonso J, Fuentes A. Loxoscelismo Cutáneo. Rev Clín Med Fam. 2012; 5(1):73-75 73. https://doi. org/10.4321/S1699-695X2012000100015

5. Moranchel L, Pineda L, Casarrubias M, Mendoza S, Olvera A, Alfaro J, Iniestra F, Briceño F. Evolución clínica de pacientes con loxoscelismo sistémico y dermonecrótico en un hospital de tercer nivel. Med Int Méx. 2017; 33(1):18-27. https:// medicinainterna.org.mx/article/evolucion-clinica-de-pacientes-con-loxoscelismo-sistemico-y-dermonecrotico-enun-hospital-de-tercer-nivel/ 
6. Swanson D, Vetter R. loxocelism. Clinics in Dermatology. 2006; 24:213-221. https://doi.org/10.1016/j. clindermatol.2005.11.006

7. Meiri O, Bacila Y, Bertoni R, Tomab L, Kalapothakisc E, Chavez E, Carlos O, Gremski W, von Dietrich C, Nader H, Sanches S. Brown spider dermonecrotic toxin directly induces nephrotoxicity. Toxicology and Applied Pharmacology. 2006; 11:64-77. https://doi.org/10.1016/j.taap.2005.05.015

8. Deboni P. Avaliação da atividade biológica induzida por fosfolipases-d recombinantes de aranha-marrom (gênero Loxosceles): ênfase no efeito sobre a membrana plasmática de células endoteliais. Monografía de conclusión de curso. Sector de Ciencias Biológicas. Universidad Federal de Paraná. 2016. Disponible desde internet en: URL: https:// acervodigital.ufpr.br/bitstream/handle/1884/46506/TCC\%20Paula\%20Deboni.pdf?sequence=1\&isAllowed=y

9. Herrera T. Guia para el Manejo de Mordedura de Araña de los Rincones - Loxosceles laeta. Departamento de Enfermedades Transmisibles División de Prevención y Control de Enfermedades: Ministerio de Salud. Chile; 2016. https://www.minsal.cl/wp-content/uploads/2016/11/LOXOSCELES-FINAL.pdf

10. Espinosa Á, Olarte M, Rofriguez C, Roncancio G. Caso sospechoso de envenenamiento por araña reclusa (Loxosceles) y revisión de la literatura. Act Colomb Cuid Int. 2014; 14(4):295-307 https://pure.urosario.edu.co/es/publications/ caso-sospechoso-de-envenenamiento-por-ara\%C3\%B1a-reclusa-loxosceles-y-

11. Manriquez J, Silva S. Loxoscelismo cutáneo y cutáneo-visceral: Revisión sistemática. Rev Chil Infect. 2009; 26(5):420432. https://doi.org/10.4067/S0716-10182009000600004

12. MRAD A. Ética en la investigación con modelos animales Experimentales. Alternativas y la 3 RS de Russel. Una responsabilidad y un compromiso ético que nos compete a todos. Rev Col Bioetica 2006; 1(1):163-184. https:// www.redalyc.org/pdf/1892/189217283010.pdf

13. Violet L, Montes D, Cardona J. Accidente ponzoñoso por arañas del género Loxosceles spp en bovinos del departamento de Córdoba. Rev Colombiana Cienc. Anim - RECIA. 2017; 9(Sup 1):55-59. https://doi.org/10.24188/ recia.v9.nS.2017.521

14. Cardona J, Buitrago J, Martínez N. Caracterización clínica del loxoscelismo dermonecrótico en equinos de Córdoba, Colombia. Rev CES Med Vet Zoot. 2017; 12(2):123-133. https://revistas.ces.edu.co/index.php/mvz/article/ view/4377

15. Escalante P, Montoya M, Terroba V, Nava A, Escalante I. Loxoscelismo local dermonecrótico en niños mordidos por la araña Loxosceles reclusa (Araña “violinista”). Gad Med Mex. 1999; 135(4):423-426. https://dialnet.unirioja.es/ servlet/articulo?codigo $=4051786$

16. De Roodt A; Salomon 0; Lloveras S; Orduna T. Envenenamiento por arañas del género Loxosceles. Medicina (Buenos Aires). 2002; 62:83-94. http://sgc.anlis.gob.ar/bitstream/123456789/68/1/Medicina\%20\%28Buenos\%20 Aires\%29\%2c\%202002\%2c\%2062\%281\%29\%2c\%2083\%e2\%80\%9394..pdf

17. Cardona J. Atlas de Dermatología Tropical en Grandes Animales. Fondo Editorial de la Universidad de Córdoba. Universidad de Córdoba. Montería, Colombia. 2018. https://www.facebook.com/1260661470723665/ posts/1675915689198239/ 